\title{
Performance Analysis of Image Compression Using Wavelets
}

\author{
Sonja Grgic, Mislav Grgic, Member, IEEE, and Branka Zovko-Cihlar, Member, IEEE
}

\begin{abstract}
The aim of this paper is to examine a set of wavelet functions (wavelets) for implementation in a still image compression system and to highlight the benefit of this transform relating to today's methods. The paper discusses important features of wavelet transform in compression of still images, including the extent to which the quality of image is degraded by the process of wavelet compression and decompression. Image quality is measured objectively, using peak signal-to-noise ratio or picture quality scale, and subjectively, using perceived image quality. The effects of different wavelet functions, image contents and compression ratios are assessed. A comparison with a discrete-cosine-transform-based compression system is given. Our results provide a good reference for application developers to choose a good wavelet compression system for their application.
\end{abstract}

Index Terms-Discrete cosine transforms, image coding, transform coding, wavelet transforms.

\section{INTRODUCTION}

$\mathbf{I}$ $\mathrm{N}$ RECENT years, many studies have been made on wavelets. An excellent overview of what wavelets have brought to the fields as diverse as biomedical applications, wireless communications, computer graphics or turbulence, is given in [1]. Image compression is one of the most visible applications of wavelets. The rapid increase in the range and use of electronic imaging justifies attention for systematic design of an image compression system and for providing the image quality needed in different applications.

A typical still image contains a large amount of spatial redundancy in plain areas where adjacent picture elements (pixels, pels) have almost the same values. It means that the pixel values are highly correlated [2]. In addition, a still image can contain subjective redundancy, which is determined by properties of a human visual system (HVS) [3]. An HVS presents some tolerance to distortion, depending upon the image content and viewing conditions. Consequently, pixels must not always be reproduced exactly as originated and the HVS will not detect the difference between original image and reproduced image. The redundancy (both statistical and subjective) can be removed to achieve compression of the image data. The basic measure for the performance of a compression algorithm is compression ratio $(\mathrm{CR})$, defined as a ratio between original data size and

Manuscript received February 27, 2000; revised November 26, 2000. Abstract published on the Internet February 15, 2001. This paper was presented at the IEEE International Symposium on Industrial Electronics, Bled, Slovenia, July $11-16,1999$.

The authors are with the Department of Radiocommunications and Microwave Engineering, Faculty of Electrical Engineering and Computing, University of Zagreb, HR-10000 Zagreb, Croatia (e-mail: sonja.grgic@ fer.hr). Publisher Item Identifier S 0278-0046(01)03379-2. compressed data size. In a lossy compression scheme, the image compression algorithm should achieve a tradeoff between compression ratio and image quality [4]. Higher compression ratios will produce lower image quality and vice versa. Quality and compression can also vary according to input image characteristics and content.

Transform coding is a widely used method of compressing image information. In a transform-based compression system two-dimensional (2-D) images are transformed from the spatial domain to the frequency domain. An effective transform will concentrate useful information into a few of the low-frequency transform coefficients. An HVS is more sensitive to energy with low spatial frequency than with high spatial frequency. Therefore, compression can be achieved by quantizing the coefficients, so that important coefficients (low-frequency coefficients) are transmitted and the remaining coefficients are discarded. Very effective and popular ways to achieve compression of image data are based on the discrete cosine transform (DCT) and discrete wavelet transform (DWT).

Current standards for compression of still (e.g., JPEG [5]) and moving images (e.g., MPEG-1 [6], MPEG-2 [7]) use DCT, which represents an image as a superposition of cosine functions with different discrete frequencies [8]. The transformed signal is a function of two spatial dimensions, and its components are called DCT coefficients or spatial frequencies. DCT coefficients measure the contribution of the cosine functions at different discrete frequencies. DCT provides excellent energy compaction, and a number of fast algorithms exist for calculating the DCT. Most existing compression systems use square DCT blocks of regular size [5]-[7]. The image is divided into blocks of $N \times N$ samples and each block is transformed independently to give $N \times N$ coefficients. For many blocks within the image, most of the DCT coefficients will be near zero. DCT in itself does not give compression. To achieve the compression, DCT coefficients should be quantized so that the near-zero coefficients are set to zero and the remaining coefficients are represented with reduced precision that is determined by quantizer scale. The quantization results in loss of information, but also in compression. Increasing the quantizer scale leads to coarser quantization, which gives high compression and poor decoded image quality.

The use of uniformly sized blocks simplified the compression system, but it does not take into account the irregular shapes within real images. The block-based segmentation of source image is a fundamental limitation of the DCT-based compression system [9]. The degradation is known as the "blocking effect" and depends on block size. A larger block leads to more 


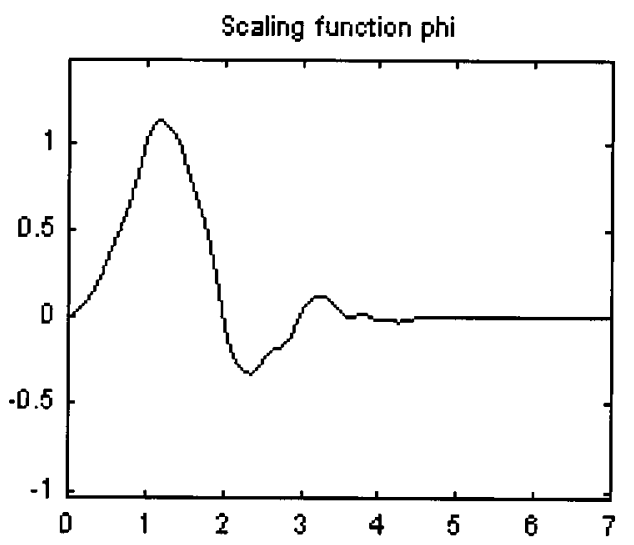

Fig. 1. Scaling and wavelet function.

efficient coding, but requires more computational power. Image distortion is less annoying for small than for large DCT blocks, but coding efficiency tends to suffer. Therefore, most existing systems use blocks of $8 \times 8$ or $16 \times 16$ pixels as a compromise between coding efficiency and image quality.

In recent times, much of the research activities in image coding have been focused on the DWT, which has become a standard tool in image compression applications because of their data reduction capability [10]-[12]. In a wavelet compression system, the entire image is transformed and compressed as a single data object rather than block by block as in a DCT-based compression system. It allows a uniform distribution of compression error across the entire image. DWT offers adaptive spatial-frequency resolution (better spatial resolution at high frequencies and better frequency resolution at low frequencies) that is well suited to the properties of an HVS. It can provide better image quality than DCT, especially on a higher compression ratio [13]. However, the implementation of the DCT is less expensive than that of the DWT. For example, the most efficient algorithm for 2-D $8 \times 8$ DCT requires only 54 multiplications [14], while the complexity of calculating the DWT depends on the length of wavelet filters.

A wavelet image compression system can be created by selecting a type of wavelet function, quantizer, and statistical coder. In this paper, we do not intend to give a technical description of a wavelet image compression system. We used a few general types of wavelets and compared the effects of wavelet analysis and representation, compression ratio, image content, and resolution to image quality. According to this analysis, we show that searching for the optimal wavelet needs to be done taking into account not only objective picture quality measures, but also subjective measures. We highlight the performance gain of the DWT over the DCT. Quantizers for the DCT and wavelet compression systems should be tailored to the transform structure, which is quite different for the DCT and the DWT. The representative quantizer for the DCT is a uniform quantizer in baseline JPEG [5], and for the DWT, it is Shapiro's zerotree quantizer [15], [16]. Hence, we did not take into account the influence of the quantizer and entropy coder, in order to accurately characterize the difference of compression performance due to the transforms (wavelet versus DCT).

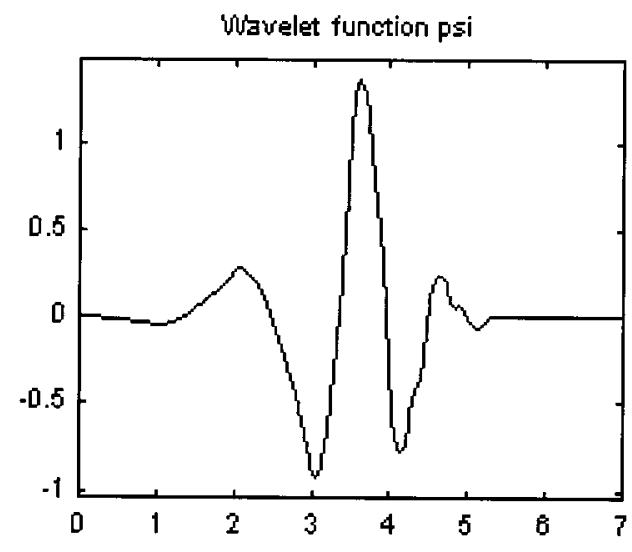

\section{WAVELET TRANSFORM}

Wavelet transform (WT) represents an image as a sum of wavelet functions (wavelets) with different locations and scales [17]. Any decomposition of an image into wavelets involves a pair of waveforms: one to represent the high frequencies corresponding to the detailed parts of an image (wavelet function $\Psi$ ) and one for the low frequencies or smooth parts of an image (scaling function $\Phi$ ).

Fig. 1 shows two waveforms of a family discovered in the late 1980s by Daubechies: the right one can be used to represent detailed parts of the image and the left one to represent smooth parts of the image. The two waveforms are translated and scaled on the time axis to produce a set of wavelet functions at different locations and on different scales. Each wavelet contains the same number of cycles, such that, as the frequency reduces, the wavelet gets longer. High frequencies are transformed with short functions (low scale). Low frequencies are transformed with long functions (high scale). During computation, the analyzing wavelet is shifted over the full domain of the analyzed function. The result of WT is a set of wavelet coefficients, which measure the contribution of the wavelets at these locations and scales.

\section{A. Multiresolution Analysis}

WT performs multiresolution image analysis [18]. The result of multiresolution analysis is simultaneous image representation on different resolution (and quality) levels [19]. The resolution is determined by a threshold below which all fluctuations or details are ignored. The difference between two neighboring resolutions represents details. Therefore, an image can be represented by a low-resolution image (approximation or average part) and the details on each higher resolution level. Let us consider a one-dimensional (1-D) function $f(t)$. At the resolution level $j$, the approximation of the function $f(t)$ is $f_{j}(t)$. At the next resolution level $j+1$, the approximation of the function $f(t)$ is $f_{j+1}(t)$. The details denoted by $d_{j}(t)$ are included in $f_{j+1}(t): f_{j+1}(t)=f_{j}(t)+d_{j}(t)$. This procedure can be repeated several times and function $f(t)$ can be viewed as

$$
f(t)=f_{j}(t)+\sum_{k=j}^{n} d_{k}(t) .
$$




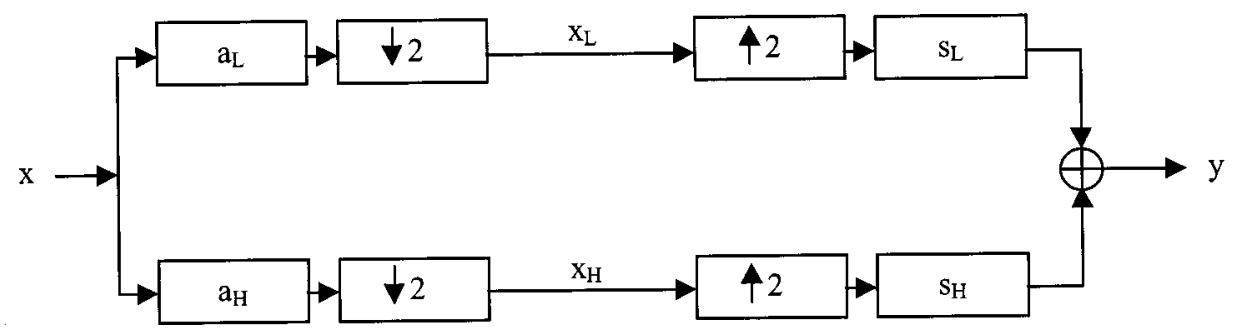

Fig. 2. Two-channel filter bank.

Similarly, the space of square integrable functions $L^{2}(R)$ can be viewed as a composition of scaling subspaces $V_{j}$ and wavelet subspaces $W_{j}$ such that the approximation of $f(t)$ at resolution $j\left(f_{j}(t)\right)$ is in $V_{j}$ and the details $d_{j}(t)$ are in $W_{j} . V_{j}$ and $W_{j}$ are defined in terms of dilates and translates of scaling function $\Phi$ and wavelet function $\Psi: V_{j}=\left\{\Phi\left(2^{j} x-k\right) \mid k \in Z\right\}$ and $W_{j}=\left\{\Psi\left(2^{j} x-k\right) \mid k \in Z\right\} . V_{j}$ and $W_{j}$ are localized in dyadically scaled frequency "octaves" by the scale or resolution parameter $2^{j}$ (dyadic scales are based on powers of two) and localized spatially by translation $k$. The scaling subspace $V_{j}$ must be contained in all subspaces on higher resolutions $\left(V_{j} \subset V_{j+1}\right)$. The wavelet subspaces $W_{j}$ fill the gaps between successive scales: $V_{j+1}=V_{j} \oplus W_{j}$. We can start with an approximation on some scale $V_{0}$ and then use wavelets to fill in the missing details on finer and finer scales. The finest resolution level includes all square integrable functions

$$
L^{2}(R)=V_{0}+\bigoplus_{j=0}^{\infty} W_{j}
$$

Since $\Phi \in V_{0} \subset V_{1}$, it follows that the scaling function for multiresolution approximation can be obtained as the solution to a two-scale dilational equation

$$
\Phi(x)=\sum_{k} a_{L}(k) \Phi(2 x-k)
$$

for some suitable sequence of coefficients $a_{L}(k)$. Once $\Phi$ has been found, an associated mother wavelet is given by a similarlooking formula

$$
\Psi(x)=\sum_{k} a_{H}(k) \Phi(2 x-k) .
$$

Some effort is required to produce appropriate coefficient sequences $a_{L}(k)$ and $a_{H}(k)$ [17].

\section{B. Discrete Wavelet Transform}

One of the big discoveries for wavelet analysis was that perfect reconstruction filter banks could be formed using the coefficient sequences $a_{L}(k)$ and $a_{H}(k)$ (Fig. 2). The input sequence $x$ is convolved with high-pass (HPF) and low-pass (LPF) filters $a_{H}(k)$ and $a_{L}(k)$ and each result is downsampled by two, yielding the transform signals $x_{H}$ and $x_{L}$. The signal is reconstructed through upsampling and convolution with high and low synthesis filters $s_{H}(k)$ and $s_{L}(k)$. For properly designed filters, the signal $x$ is reconstructed exactly $(y=x)$.
The choice of filter not only determines whether perfect reconstruction is possible, it also determines the shape of wavelet we use to perform the analysis. By cascading the analysis filter bank with itself a number of times, a digital signal decomposition with dyadic frequency scaling known as DWT can be formed. The mathematical manipulation that effects synthesis is called inverse DWT. An efficient way to implement this scheme using filters was developed by Mallat [19]. The new twist that wavelets bring to filter banks is connection between multiresolution analysis (that, in principle, can be performed on the original, continuous signal) and digital signal processing performed on discrete, sampled signals.

DWT for an image as a 2-D signal can be derived from 1-D DWT. The easiest way for obtaining scaling and wavelet function for two dimensions is by multiplying two 1-D functions. The scaling function for 2-D DWT can be obtained by multiplying two 1-D scaling functions: $\Phi(x, y)=\Phi(x) \Phi(y)$. Wavelet functions for 2-D DWT can be obtained by multiplying two wavelet functions or wavelet and scaling function for 1-D analysis. For the 2-D case, there exist three wavelet functions that scan details in horizontal $\Psi^{(I)}(x, y)=\Phi(x) \Psi(y)$, vertical $\Psi^{(I I)}(x, y)=\Psi(x) \Phi(y)$, and diagonal directions: $\Psi^{(I I I)}(x, y)=\Psi(x) \Psi(y)$. This may be represented as a four-channel perfect reconstruction filter bank as shown in Fig. 3. Now, each filter is 2-D with the subscript indicating the type of filter (HPF or LPF) for separable horizontal and vertical components. The resulting four transform components consist of all possible combinations of high- and low-pass filtering in the two directions. By using these filters in one stage, an image can be decomposed into four bands. There are three types of detail images for each resolution: horizontal (HL), vertical (LH), and diagonal ( $\mathrm{HH})$. The operations can be repeated on the low-low band using the second stage of identical filter bank. Thus, a typical 2-D DWT, used in image compression, will generate the hierarchical pyramidal structure shown in Fig. 3(b). Here, we adopt the term "number of decompositions" $(J)$ to describe the number of 2-D filter stages used in image decomposition.

Wavelet multiresolution and direction selective decomposition of images is matched to an HVS [20]. In the spatial domain, the image can be considered as a composition of information on a number of different scales. A wavelet transform measures gray-level image variations at different scales. In the frequency domain, the contrast sensitivity function of the HVS depends on frequency and orientation of the details. 


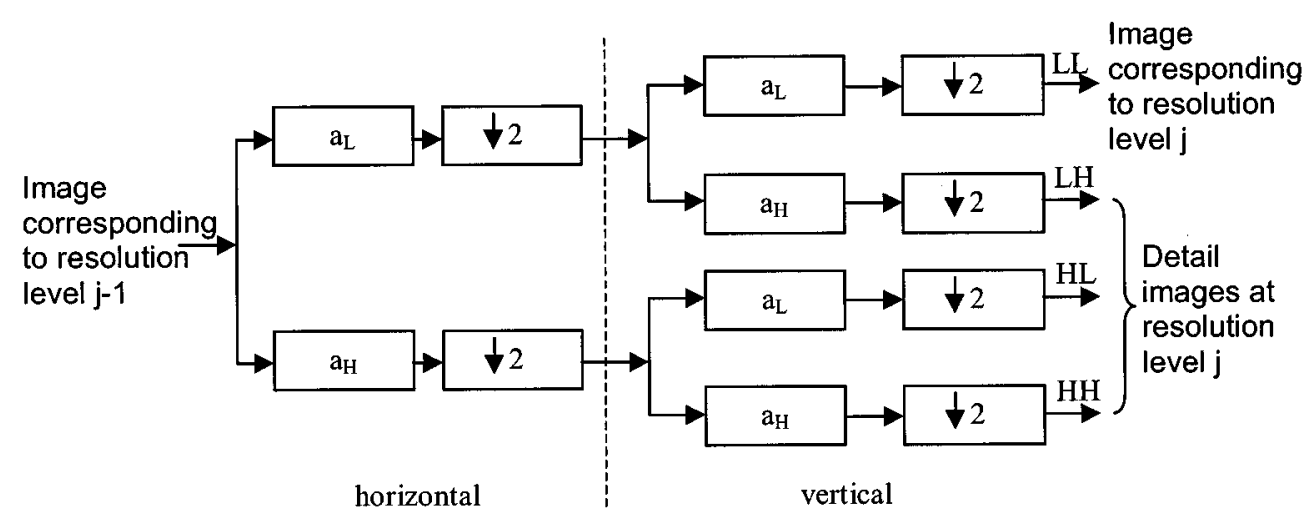

(a)

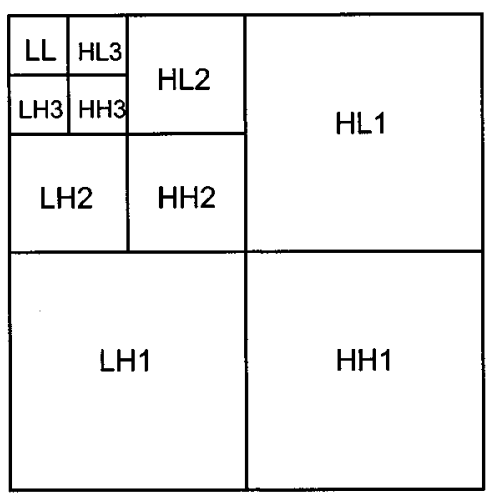

(b)

Fig. 3. (a) One filter stage in 2-D DWT. (b) Pyramidal structure of a wavelet decomposition.

\section{IMAGE QUALITy EVALUATION}

The image quality can be evaluated objectively and subjectively [21]. Objective methods are based on computable distortion measures. A standard objective measure of image quality is reconstruction error. Suppose that one has a system in which an input image element block $\{x(n)\}, n=0,1, \ldots, N-1$ is reproduced as $\{y(n)\}, n=0,1, \ldots, N-1$. The reconstruction error $r(n)$ is defined as the difference between $x(n)$ and $y(n)$

$$
r(n)=x(n)-y(n) .
$$

The variances of $x(n), y(n)$, and $r(n)$ are $\sigma_{x}^{2}, \sigma_{y}^{2}$, and $\sigma_{r}^{2}$. In the special case of zero-means signals, variances are simply equal to respective mean-square values over appropriate sequence length $M$

$$
\sigma_{z}^{2}=\frac{1}{M} \sum_{n=1}^{M} z^{2}(n), \quad z=x, y, \text { or } r .
$$

A standard objective measure of coded image quality is signal-to-noise ratio (SNR) which is defined as the ratio between signal variance and reconstruction error variance [mean-square error (MSE)] usually expressed in decibels (dB)

$$
\mathrm{SNR}(\mathrm{dB})=10 \log _{10}\left(\frac{\sigma_{x}^{2}}{\sigma_{r}^{2}}\right)=10 \log _{10}\left(\frac{\sigma_{x}^{2}}{\operatorname{MSE}}\right)
$$

When the input signal is an $R$-bit discrete variable, the variance or energy can be replaced by the maximum input symbol energy $\left(2^{R}-1\right)^{2}$. For the common case of 8 bits per picture element of input image, the peak SNR (PSNR) can be defined as

$$
\operatorname{PSNR}(\mathrm{dB})=10 \log _{10}\left(\frac{255^{2}}{\mathrm{MSE}}\right) .
$$

SNR is not adequate as a perceptually meaningful measure of picture quality, because the reconstruction errors in general do not have the character of signal-independent additive noise, and the seriousness of the impairments cannot be measured by a simple power measurement [22]. Small impairment of an image can lead to a very large value of $\sigma_{r}^{2}$ and, consequently, a very small value of PSNR, in spite of the fact that the perceived image quality can be very acceptable. In fact, in image compression systems, the truly definitive measure of image quality is perceptual quality. The distortion is specified by mean opinion score (MOS) [23] or by picture quality scale (PQS) [24].

In addition to the commonly used PSNR, we chose to use a perception based subjective evaluation, quantified by MOS, and a perception-based objective evaluation, quantified by PQS. For the set of distorted images, the MOS values were obtained from an experiment involving 11 viewers. The viewers were allowed to give half-scale grades. The testing methodology was the double-stimulus impairment scale method with five-grade impairment scale described in [25]. When the tests span the full 


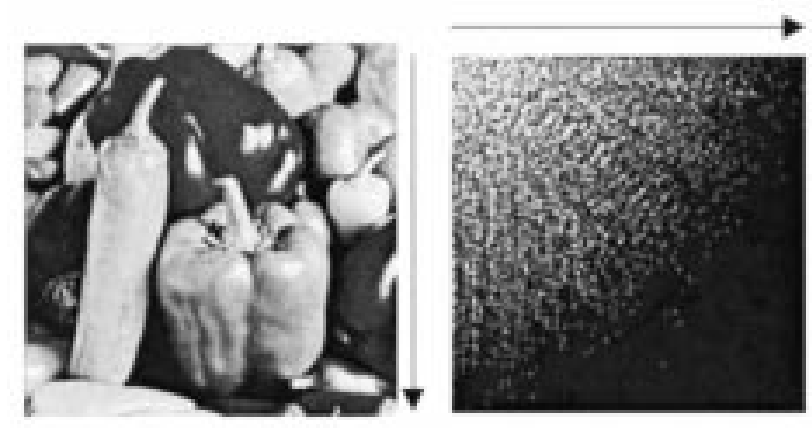

(a)
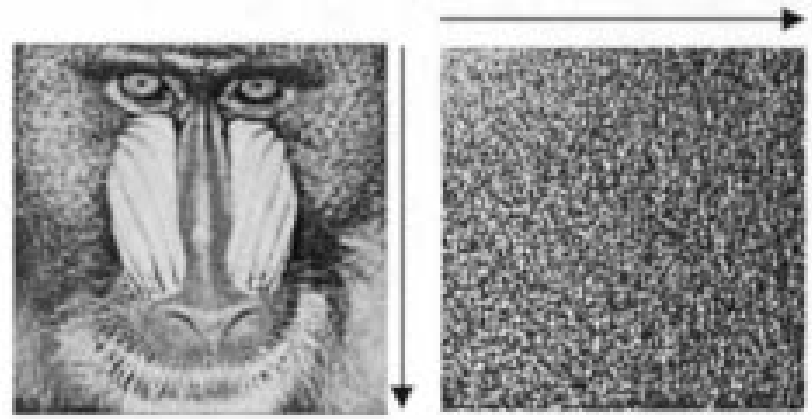

(c)
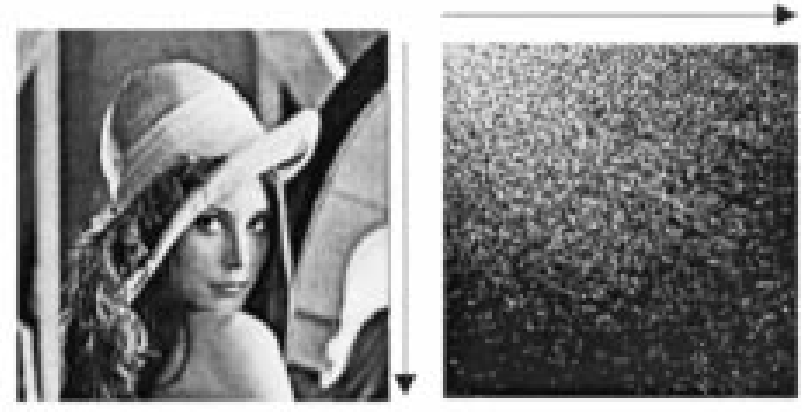

(b)
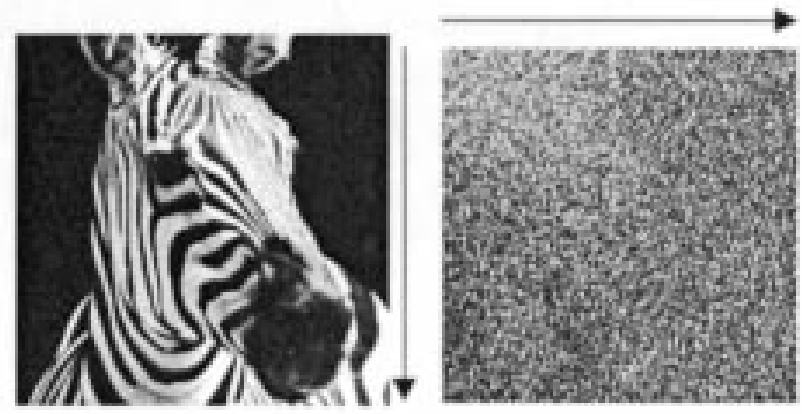

(d)

Fig. 4. Frequency content of test images. (a) Peppers. (b) Lena. (c) Baboon. (d) Zebra.

range of impairments (as in our experiment), the double-stimulus impairment scale method is appropriate and should be used.

The double-stimulus impairment scale method uses reference and test conditions which are arranged in pairs such that the first in the pair is the unimpaired reference and the second is the same sequence impaired. The original source image without compression was used as the reference condition. The assessor is asked to vote on the second, keeping in mind the first. The method uses the five-grade impairment scale with proper description for each grade: 5-imperceptible; 4-perceptible, but not annoying; 3 -slightly annoying; 2-annoying; and 1-very annoying. At the end of the series of sessions, MOS for each test condition and test image are calculated

$$
\operatorname{MOS}=\sum_{i=1}^{5} i \cdot p(i)
$$

where $i$ is grade and $p(i)$ is grade probability.

Subjective assessments of image quality are experimentally difficult and lengthy, and the results may vary depending on the test conditions. In addition to MOS, we used PQS methodology proposed in [24], [26]. The PQS has been developed in the last few years for evaluating the quality of compressed images. It combines various perceived distortions into a single quantitative measure. To do so, PQS methodology uses some of the properties of HVS relevant to global image impairments, such as random errors, and emphasize the perceptual importance of structured and localized errors. PQS is constructed by regressions with MOS, which is a five-level grading scale. PQS closely approximates the MOS in the middle of the quality range. For very-high-quality images, it is possible to obtain values of PQS larger than 5 . At the low end of the image quality scale, $\mathrm{PQS}$ can obtain negative values (meaningless results). It was the reason that we had to use subjective evaluation (our test includes low quality images) but PQS helped us in some phases of our research work.

\section{DWT IN IMAGE COMPRESSION}

\section{A. Image Content}

The fundamental difficulty in testing an image compression system is how to decide which test images to use for the evaluations. The image content being viewed influences the perception of quality irrespective of technical parameters of the system [9]. Normally, a series of pictures, which are average in terms of how difficult they are for system being evaluated, has been selected. To obtain a balance of critical and moderately critical material we used four types of test images with different frequency content: Peppers, Lena, Baboon, and Zebra. Spectral activity of test images is evaluated using DCT applied to the whole image. DCT coefficients as a result of DCT show frequency content of the image. Fig. 4 shows the distributions of image values before and after DCT. The distribution of DCT coefficients depends on image content (white dots represent DCT coefficients, arrows indicate the increase of horizontal and vertical frequency). Moving across the top row, horizontal spatial frequency increases. Moving down, vertical spatial frequency increases. Images with high spectral activity are more difficult for a compression system to handle. These images usually contain large number of small details and low spatial redundancy.

Choice of wavelet function is crucial for coding performance in image compression [27]. However, this choice should be adjusted to image content [28]. The compression performance for images with high spectral activity is fairly insensitive to choice 
of compression method (for example, test image Baboon), [32]. On the other hand, coding performance for images with moderate spectral activity (for example, test image Lena) are more sensitive to choice of compression method. The best way for choosing wavelet function is to select optimal basis for images with moderate spectral activity. This wavelet will give satisfying results for other types of images.

\section{B. Choice of Wavelet Function}

Important properties of wavelet functions in image compression applications are compact support (lead to efficient implementation), symmetry (useful in avoiding dephasing in image processing), orthogonality (allow fast algorithm), regularity, and degree of smoothness (related to filter order or filter length).

In our experiment, four types of wavelet families are examined: Haar Wavelet (HW), Daubechies Wavelet (DW), Coiflet Wavelet $(\mathrm{CW})$, and Biorthogonal Wavelet (BW). Each wavelet family can be parameterized by integer $N$ that determines filter order. Biorthogonal wavelets can use filters with similar or dissimilar orders for decomposition $(N d)$ and reconstruction $(N r)$. In our examples, different filter orders are used inside each wavelet family. We have used the following sets of wavelets: DW- $N$ with $N=1,2,3,4,5,6,10$ [17], CW- $N$ with $N=2,3,4,5$ [17], and BW- $N r . N d$ with $(N r, N d)=(1,3),(2,2),(3,1),(3,3),(3,5),(6.8),[30]$. Daubechies and Coiflet wavelets are families of orthogonal wavelets that are compactly supported. Compactly supported wavelets correspond to finite-impulse response (FIR) filters and, thus, lead to efficient implementation [29]. Only ideal filters with infinite duration can provide alias-free frequency split and perfect interband decorrelation of coefficients. Since time localization of the filter is very important in visual signal processing, arbitrarily long filters cannot be used. A major disadvantage of DW and CW is their asymmetry, which can cause artifacts at borders of the wavelet subbands. DW is asymmetrical while $\mathrm{CW}$ is almost symmetrical. Symmetry in wavelets can be obtained only if we are willing to give up either compact support or orthogonality of wavelet (except for HW, which is orthogonal, compactly supported, and symmetric). If we want both symmetry and compact support in wavelets, we should relax the orthogonality condition and allow nonorthogonal wavelet functions. An example is the family of biorthogonal wavelets that contains compactly supported and symmetric wavelets [30].

\section{Filter Order and Filter Length}

The filter length $L$ is determined by filter order, but relationship between filter order and filter length is different for different wavelet families. For example, the filter length is $L=$ $2 \cdot N$ for the DW family and $L=6 \cdot N$ for the CW family. HW is the special case of DW with $N=1$ (DW-1) and $L=2$. Filter lengths are approximately $L=\{\max (2 N d, 2 N r)+2\}$, but effective lengths are different for LPF and HPF used for decomposition and reconstruction and should be determined for each filter type. Fig. 5 shows examples of scaling and wavelet functions from each wavelet family. Filter coefficients for some of the examples from Fig. 5 are given in Table I. In DW, family scaling and wavelet functions for different filter orders have different shapes [Fig. 5(a)-(d)]. Scaling and wavelet functions in the CW family for all filter orders have very similar shapes [Fig. 5(e) and (f)]. Scaling and wavelet functions for decomposition and reconstruction in the BW family can be similar or dissimilar. BW-2.2 is such that decomposition and reconstruction functions have different shapes [Fig. 5(g) and (h)], but for BW-6.8 these functions are very close to each other [Fig. 5(i) and (j)].

Higher filter orders give wider functions in the time domain with higher degree of smoothness. Filter with a high order can be designed to have good frequency localization, which increases the energy compaction. Wavelet smoothness also increases with its order. Filters with lower order have a better time localization and preserve important edge information. Wavelet-based image compression prefers smooth functions (that can be achieved using long filters) but complexity of calculating DWT increases by increasing the filter length. Therefore, in image compression application we have to find balance between filter length, degree of smoothness, and computational complexity. Inside each wavelet family, we can find wavelet function that represents optimal solution related to filter length and degree of smoothness, but this solution depends on image content.

\section{Number of Decompositions}

The quality of compressed image depends on the number of decompositions $(J)$. The number of decompositions determines the resolution of the lowest level in wavelet domain. If we use larger number of decompositions, we will be more successful in resolving important DWT coefficients from less important coefficients. The HVS is less sensitive to removal of smaller details [31].

After decomposing the image and representing it with wavelet coefficients, compression can be performed by ignoring all coefficients below some threshold. In our experiment, compression is obtained by wavelet coefficient thresholding using a global positive threshold value. All coefficients below some threshold are neglected and compression ratio is computed. Compression algorithm provides two modes of operation: 1) compression ratio is fixed to the required level and threshold value has been changed to achieve required compression ratio; after that, PSNR is computed; 2) PSNR is fixed to the required level and threshold values has been changed to achieve required PSNR; after that, CR is computed.

Fig. 6 shows comparison of reconstructed image Lena $(256 \times$ 256 pixels, 8 bit/pixel) for 1, 2, 3, and 4 decompositions (CR $=$ $50: 1$ ). In this example, DW-5 is used. It can be seen that image quality is better for a larger number of decompositions. On the other hand, a larger number of decompositions causes the loss of the coding algorithm efficiency. Therefore, adaptive decomposition is required to achieve balance between image quality and computational complexity. PSNR tends to saturate for a larger number of decompositions [Fig. 7(a)]. For each compression ratio, the PSNR characteristic has "threshold" which represents the optimal number of decompositions. Below and above the threshold, PSNR decreases. For DW-5 used in this example optimal number of decompositions is 5 [Fig. 7(b)]. 

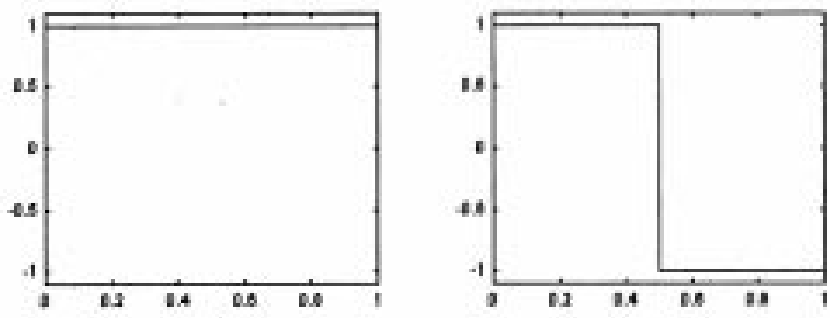

(a)
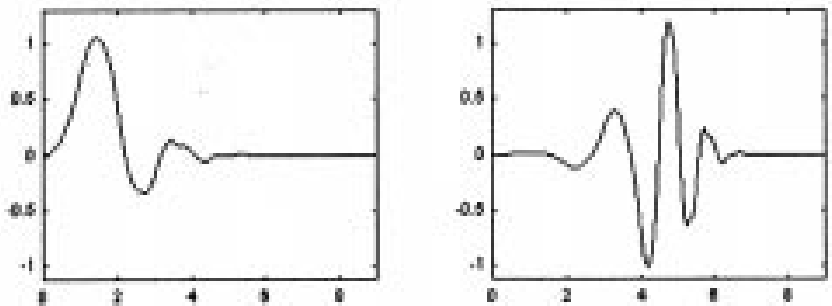

(c)
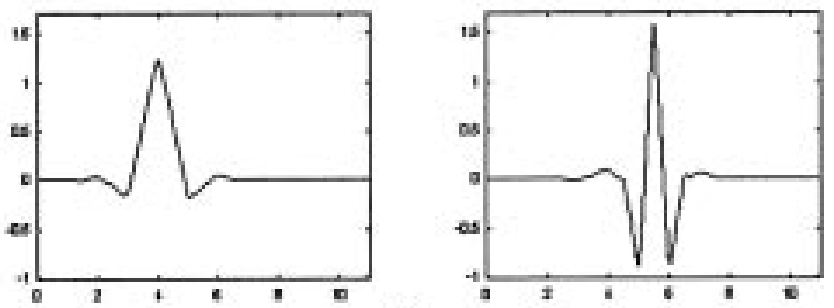

(e)
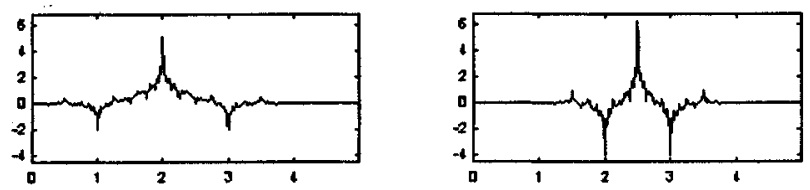

(g)
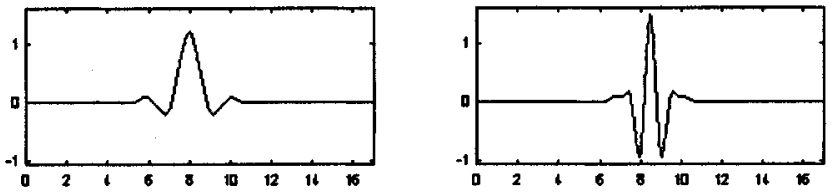

(i)
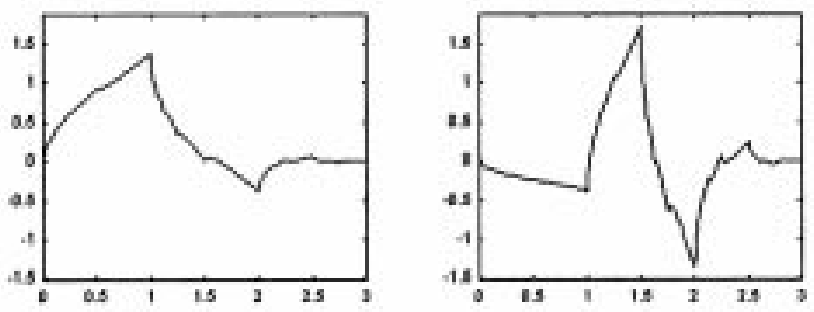

(b)
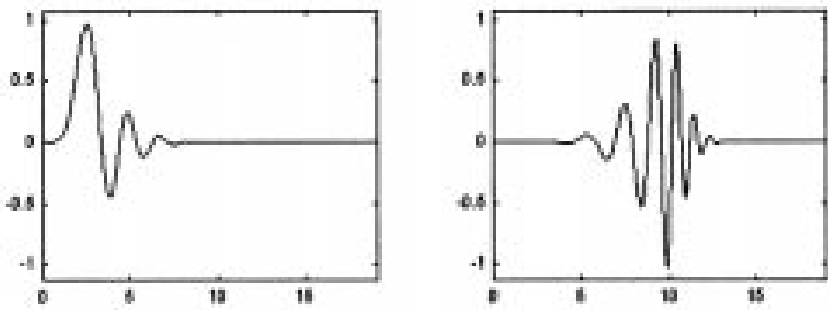

(d)
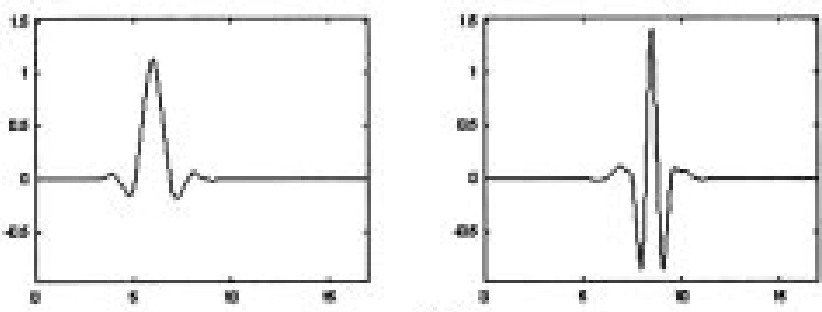

(f)
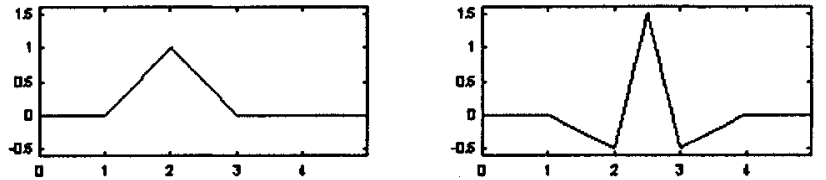

(h)
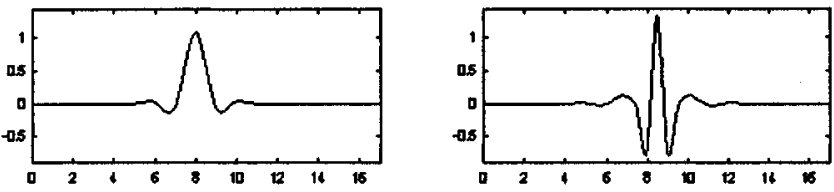

(j)

Fig. 5. Scaling and wavelet functions for different wavelet families. (a) DW-1, $N=1, L=2$. (b) DW-2, $N=2, L=4$. (c) DW-5, $N=5, L=10$. (d) DW-10, $N=10, L=20$. (e) CW-2, $N=2, L=12$. (f) CW-3, $N=3, L=18$. (g) BW-2.2 for decomp, $N=2, L(\mathrm{LPF})=5, L(\mathrm{HPF})=3$. (h) BW-2.2 for recons, $N=2, L(\mathrm{LPF})=3, L(\mathrm{HPF})=5$. (i) BW-6.8 for decomp, $N=6, L(\mathrm{LPF})=17, L$ (HPF) $=11$. (j) BW-6.8 for recons, $N=8, L(\mathrm{LPF})=11, L(\mathrm{HPF})=17$.

The optimal number of decompositions depends on filter order. Fig. 7(b) shows PSNR values for different filter orders and fixed compression ratio $(10: 1)$. It can be seen that as the number of decompositions increases, PSNR is increased up to some number of decompositions. Beyond that, increasing the number of decompositions has a negative effect. Higher filter order [for example, DW-10 in Fig. 7(b)] does not imply better image quality because of the filter length, which becomes the limiting factor for decomposition. Decisions about the filter order and number of decompositions are a matter of compromise. Higher order filters give broader function in the time domain. On the other hand, the number of decompositions determines the resolution of the lowest level in wavelet domain. If the order of function gives a time window of function larger than the time interval needed for analysis of lowest level, the picture quality can only degrade.

\section{E. Computational Complexity}

Computational complexity of the wavelet transform for an image size of $M \times M$ employing dyadic decomposition is approximately [32]

$$
C=16 \cdot M^{2} \cdot L \cdot\left(1-4^{-J}\right) / 3
$$


TABLE I

Filter COEFFICIENTS FOR DW-1, DW-2, DW-5, CW-2, CW-3, BW-2.2, AND BW-6.8

\begin{tabular}{|c|c|c|c|c|c|c|c|c|c|}
\hline & DW-1 & DW-2 & DW-5 & CW-2 & CW-3 & \multicolumn{2}{|c|}{ BW-2.2 } & \multicolumn{2}{|c|}{ BW-6.8 } \\
\hline k & $a_{L}(k)$ & $\mathbf{a}_{\mathrm{L}}(\mathbf{k})$ & $a_{L}(k)$ & $\mathbf{a}_{\mathrm{L}}(\mathbf{k})$ & $a_{L}(k)$ & $\mathbf{a}_{\mathbf{L}}(\mathbf{k})$ & $s_{L}(k)$ & $a_{L}(k)$ & $s_{\mathrm{L}}(\mathrm{k})$ \\
\hline 0 & 0.7071 & -0.1294 & 0.0033 & -0.0007 & -0.0000 & 0 & 0 & 0 & 0 \\
\hline 1 & 0.7071 & 0.2241 & -0.0126 & -0.0018 & -0.0001 & -0.1768 & 0.3536 & 0.0019 & 0 \\
\hline 2 & & 0.8365 & -0.0062 & 0.0056 & 0.0005 & 0.3536 & 0.7071 & -0.0019 & 0 \\
\hline 3 & & 0.4830 & 0.0776 & 0.0237 & 0.0011 & 1.0607 & 0.3536 & -0.0170 & 0.0144 \\
\hline 4 & & & -0.0322 & -0.0594 & -0.0026 & 0.3536 & 0 & 0.0119 & 0.0145 \\
\hline 5 & & & -0.2423 & -0.0765 & -0.0090 & -0.1768 & 0 & 0.0497 & -0.0787 \\
\hline 6 & & & 0.1384 & 0.4170 & 0.0159 & & & -0.0773 & -0.0404 \\
\hline 7 & & & 0.7243 & 0.8127 & 0.0346 & & & -0.0941 & 0.4178 \\
\hline 8 & & & 0.6038 & 0.3861 & -0.0823 & & & 0.4208 & 0.7589 \\
\hline 9 & & & 0.1601 & -0.0674 & -0.0718 & & & 0.8259 & 0.4178 \\
\hline 10 & & & & -0.0415 & 0.4285 & & & 0.4208 & -0.0404 \\
\hline 11 & & & & 0.0164 & 0.7938 & & & -0.0941 & -0.0787 \\
\hline 12 & & & & & 0.4052 & & & -0.0773 & 0.0145 \\
\hline 13 & & & & & -0.0611 & & & 0.0497 & 0.0144 \\
\hline 14 & & & & & -0.0658 & & & 0.0119 & 0 \\
\hline 15 & & & & & 0.0235 & & & -0.0170 & 0 \\
\hline 16 & & & & & 0.0078 & & & -0.0019 & 0 \\
\hline 17 & & & & & -0.0038 & & & 0.0019 & 0 \\
\hline
\end{tabular}

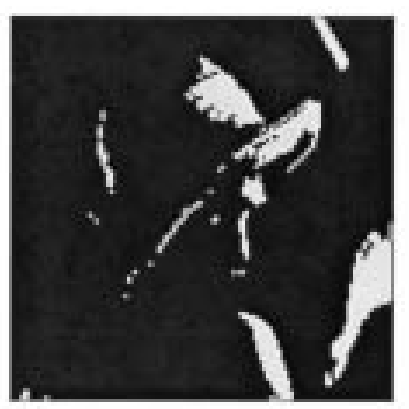

(a)

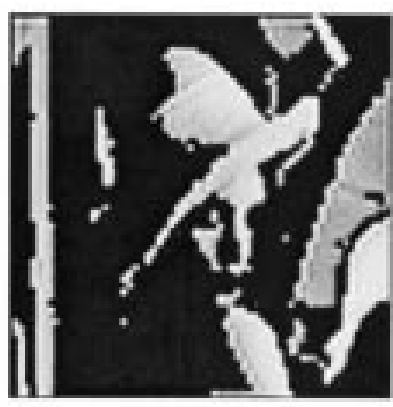

(b)

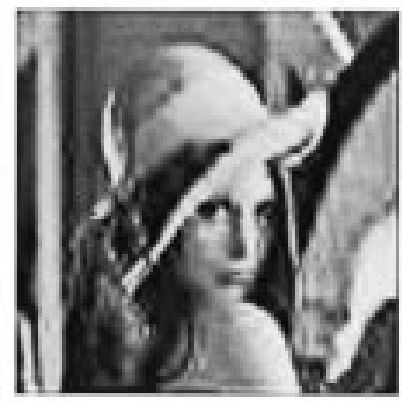

(c)

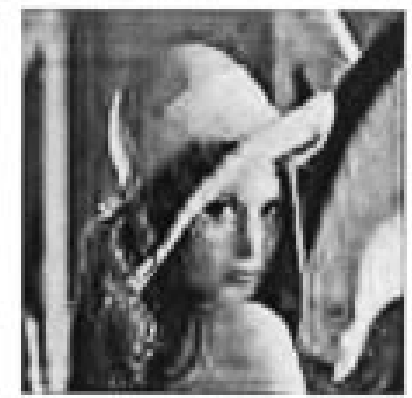

(d)

Fig. 6. Reconstructed image Lena; DW-5; CR $=50: 1$. (a) $J=1$ (PSNR $=8.40 \mathrm{~dB})$. (b) $J=2$ (PSNR $=11.76 \mathrm{~dB})$. (c) $J=3$ (PSNR $=23.39 \mathrm{~dB})$. (d) $J=4(\mathrm{PSNR}=24.40 \mathrm{~dB})$.

where $L$ and $J$ are filter length and number of decompositions, respectively. For simplicity, we consider only the computation required for calculating the wavelet transform. For example, for a $256 \times 256$ image decomposed using $J=5$ and $L=10$, the complexity will be approximately 3.5 million operations (MOP).

\section{DWT COMPRESSION RESULTS}

The choice of optimal wavelet function in an image compression system for different image types can be provided in a few steps. For each filter order in each wavelet family, the optimal number of decompositions can be found. The optimal number of decompositions gives the highest PSNR values in the wide range of compression ratios for a given filter order. Table II shows some of the results for DW and image Lena. For lower filter orders, better results are reached with more decompositions than for higher filter orders. The shaded areas in Table II show the optimal number of decompositions for a given filter order, while bold type mark the optimal combination of filter order and number of decompositions for image Lena ( 5 decompositions, filter order 5). Similar results were achieved for other wavelet families and other test images. Table III shows some of the results. For each wavelet family, different filter orders are tested using different test images. For each test image and each wavelet family, the optimal combination of filter order and number of decompositions was found (shaded areas in Table III).

The filter orders which give the best PSNR results inside each wavelet family are different for different test images, except for the BW family where filters with order 2 in decomposition and order 2 in reconstruction (BW-2.2) give the best results for all image types.

The comparison of PSNR values of optimal filters (shaded areas in Table III) from each wavelet family for different test images shows that image Peppers (low spectral activity) has the highest PSNR values and image Zebra (high spectral activity) has the smallest PSNR values. PSNR values depend on image 


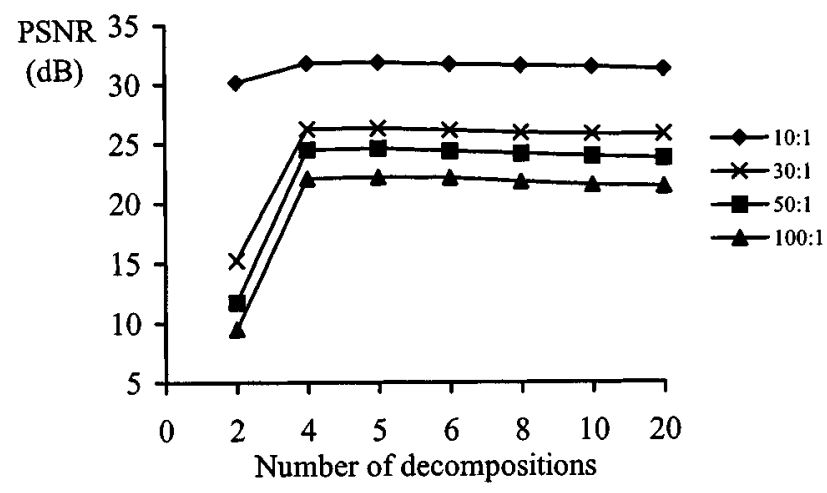

(a)

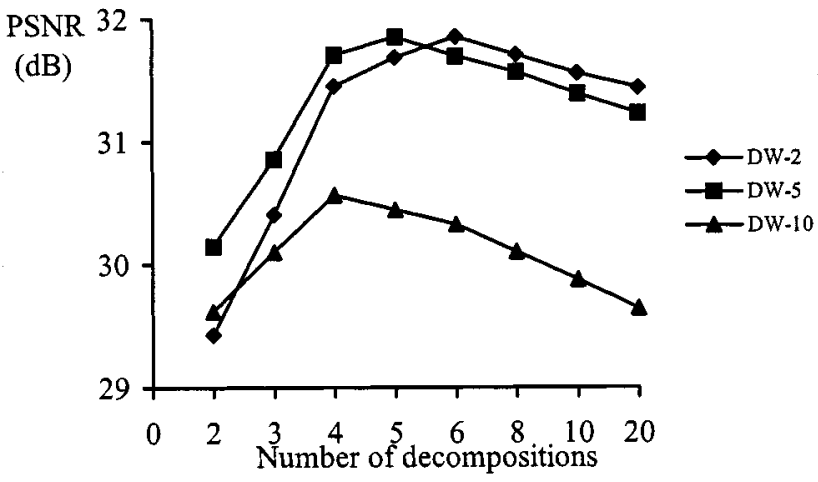

(b)

Fig. 7. PSNR for different number of decompositions. (a) DW-5. (b) $\mathrm{CR}=$ $10: 1$.

type and cannot be used if we want to compare images with different content.

Table IV compares PSNR and PQS values of optimal wavelets (shaded areas in Table III) from each wavelet family for different test images. Table III presents a rough comparison for only three compression ratios. Compression ratios 50:1 and 100:1 produce very poor image qualities that cannot be evaluated using PQS. Therefore, Table IV covers lower compression ratios that can provide useful image qualities, which can be measured using PQS. Shaded areas show the best wavelets according to the PQS values. Examination of these values reveals that BW-2.2, in most cases (14 out of 16), results in better visual quality than other wavelets for all images (BW-2.2 wavelet presents symmetric and smooth function of relatively short support).

Fig. 8 compares visual quality of image Lena compressed with optimal wavelet functions from each wavelet family. PSNR values are the same for all images ( $36 \mathrm{~dB})$, but PQS values are different. It means that comparison, which is based on PQS, shows different results than comparison based on PSNR. The best PQS results from Fig. 8 are achieved using BW-2.2. Table III shows that, for test images Zebra and Baboon, the best results are achieved using CW-2 and CW-3, but Table IV shows that the best visual quality is achieved using $\mathrm{BW}-2.2$. The last column in Table III shows computational complexity for each wavelet. It can be seen that DW-1 (HW) has the lowest computational complexity. However, DW-1 introduces a very annoying blocking effect for CR $>10: 1$. For example, PSNR results for test image Baboon show that DW-1 gives similar results as other wavelets, but with respect to visual image quality, this wavelet introduce blocking artifacts, that cannot be evaluated using PSNR (Fig. 9). Hence, we can conclude that BW-2.2 is the best choice of optimal wavelet function, not only according to visual quality (PQS), but also according to very low computational complexity (1.4 MOP).

Therefore, if we want to find the best wavelet for some image compression application, we have to take into account visual quality [34]. If we consider only the PSNR values, we can make wrong decisions. BW-2.2 provides the best visual image quality for all images. For that reason, BW-2.2 is used in further analysis and comparison with DCT.

The comparative study of wavelet coders for still images performed in [13] shows that the set partitioning in hierarchical trees (SPIHT) coder described in [16] has better performance than other coders. The SPIHT coder uses $9 / 7$ biorthogonal wavelet filter (9/7-BW) and 5 decompositions. Therefore, we implemented 9/7-BW in our compression scheme and we compared compression results with the results achieved with BW-2.2. Compression results for both wavelets are given in Table V. From Table V, we can see that PSNR values are better for 9/7-BW for all images except Peppers. On the contrary, PQS results show that BW-2.2 produces better visual picture quality, which is of higher importance for the viewer than PSNR values. Hence, the usage of BW-2.2 in the wavelet coder described in [13] can improve visual image quality. Once again, we want to emphasize that PSNR cannot be used as a definitive measure of picture quality in an image compression system. Additionally, computational complexity of $9 / 7-\mathrm{BW}$ is $2.8 \mathrm{MOP}$, that is, $2 \times$ more operations compared with $\mathrm{BW}-2.2$, which again proves that $\mathrm{BW}-2.2$ is a better choice for wavelet image compression.

\section{A. Comparative Study of $8 \times 8 D C T$ and DWT}

A comparison of PSNR values for $8 \times 8$ DCT and DWT (BW-2.2) is shown in Fig. 10. Compression results for DCT are taken from [33]. For compression ratios below 30:1, $8 \times$ 8 DCT gives similar results as DWT. For higher compression ratios $(>30: 1)$ the quality of images compressed using DWT slowly degrades, while the quality of standard DCT compressed images deteriorates rapidly. Computational complexity for $8 \times$ $8 \mathrm{DCT}$ is approximately 0.5 MOP [14]. For lower compression ratios, DCT should be used, because implementation of DCT is less expensive than that of the DWT. For the higher compression ratio (>30:1), DCT cannot be used because of very poor image quality.

For higher compression ratios, the compression performance of DWT is superior to that of $8 \times 8$ DCT and the visual quality of reconstructed images is better, even if the PSNR are the same. There are noticeable blocking artifacts in the DCT images. Fig. 11 shows the visual quality for DCT and DWT compressed images with the same PSNR (26 dB) and reconstruction error for both images. The comparison demonstrates the different nature of reconstruction error in DCT and DWT compression systems. Even for relatively high compression ratios (>30:1), DWT-based compression gives good results according to both visual quality and PSNR. 
TABLE II

OPTIMAL NUMBER OF DECOMPOSITIONS FOR DIFFERENT FILTER ORDERS IN DW FAMILY

\begin{tabular}{|c|c|c|c|c|c|c|}
\hline \multicolumn{2}{|c|}{ Wavelet } & \multicolumn{4}{|c|}{ Compression Ratio } & \multirow{2}{*}{$\frac{5}{2}$} \\
\hline Family & Order & $5: 1$ & $30: 1$ & $50: 1$ & $100: 1$ & \\
\hline \multirow{20}{*}{ DW } & \multirow{4}{*}{1} & 35.53 & 14.76 & 11.53 & 9.42 & 2 \\
\hline & & 36.29 & 25.68 & 23.79 & 21.59 & 4 \\
\hline & & 36.39 & 25.74 & 23.94 & 21.87 & 6 \\
\hline & & 36.28 & 25.75 & 23.96 & 21.94 & 8 \\
\hline & \multirow{5}{*}{2} & 36.79 & 15.09 & 11.73 & 951 & 2 \\
\hline & & 37.25 & 25,86 & 23.86 & 21.36 & 4 \\
\hline & & 37.18 & 25.79 & 23.89 & 21.52 & 6 \\
\hline & & 37.18 & 25.72 & 23.73 & 21.33 & 8 \\
\hline & & 37.07 & 25.61 & 23.57 & 21.04 & 10 \\
\hline & \multirow{6}{*}{5} & 37.04 & 15.23 & 11.73 & 9.51 & 2 \\
\hline & & 37.24 & 25.83 & 2338 & 16.16 & 3 \\
\hline & & 37,16 & 26.21 & 24.46 & 22.09 & 4 \\
\hline & & 37.05 & 26.24 & 24.53 & 22.18 & 5 \\
\hline & & 36.98 & 26.13 & 2437 & 22.13 & 6 \\
\hline & & 36.88 & 25.91 & 24.17 & 21.82 & 8 \\
\hline & \multirow{5}{*}{10} & 35.65 & 15.15 & 11.78 & 9.52 & 2 \\
\hline & & 36.00 & 25.41 & 23.20 & 16.46 & 3 \\
\hline & & 35.72 & 25.49 & 23.85 & 21.81 & 4 \\
\hline & & 35.47 & 24.13 & 23.45 & 21.41 & 6 \\
\hline & & 35.08 & 24.47 & 22.67 & 20.38 & 10 \\
\hline
\end{tabular}

TABLE III

PSNR RESUlTS IN DECIBELS FOR DIFFERENT WAVELET FAMILIES AND DIFFERENT COMPRESSION RATIOS

\begin{tabular}{|c|c|c|c|c|c|c|c|c|c|c|c|c|c|c|}
\hline \multirow{2}{*}{$\frac{\frac{E}{2}}{2}$} & \multicolumn{3}{|c|}{ Peppers } & \multicolumn{3}{|c|}{ Lena } & \multicolumn{3}{|c|}{ Zebra } & \multicolumn{3}{|c|}{ Baboon } & \multirow{2}{*}{ 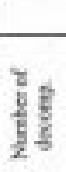 } & \multirow[b]{2}{*}{$\frac{8}{2}$} \\
\hline & 5:1 & $90-1$ & 100:t & $5: 1$ & 50.1 & 1004 & $9: 1$ & $50-1$ & 1091 & 5.1 & 50.1 & 100.1 & & \\
\hline DW-1 & 3798 & 2391 & 21.72 & 36.28 & 2356 & 21.54 & 29.16 & 17.26 & 15.9 & 23.01 & 2184 & 2100 & 8 & 0.7 \\
\hline DW.3 & 1931 & 2430 & 21.70 & 37.33 & 24.26 & 22.14 & 28.06 & 17.75 & 16.25 & 27.93 & 21.68 & 20.37) & 6 & 2.1 \\
\hline DWS & 38.75 & 2424 & 21.69 & 3705 & 24.53 & 22.18 & 27.72 & 17.81 & 16.11 & 28.21 & 21.76 & 20.77 & 5 & 35 \\
\hline $\mathrm{Dw}-10$ & 37.60 & 2306 & 1606 & 3600 & 23.20 & 16.46 & 27.92 & 1684 & 14.4 & 2759 & 21.15 & 1257 & 3 & 6.9 \\
\hline $\mathrm{CW}-1$ & 39.67 & 2462 & 22.18 & 37.80 & 24.55 & 22.33 & 28.30 & 1792 & 1644 & 28.22 & 21.82 & 20.95 & 5 & 42 \\
\hline CW:-3 & 3965 & 2489 & 22.79 & 37.73 & 20.4 & 22.49 & 25.10 & 1795 & 16.77 & 2824 & 2185 & 20.87 & 4 & 6.3 \\
\hline CW-4 & 38.96 & 2496 & 21.81 & 37.92 & 2450 & 22.15 & 2795 & 1793 & 1617 & 2823 & 21.75 & $20 . n$ & 4 & 8.4 \\
\hline CW.S & 38.92 & 24.97 & 21.94 & 37.46 & 23.54 & 22.43 & 2790 & 1797 & 16.30 & 2824 & 21.76 & 20.86 & 4 & 16.4 \\
\hline 86.22 & 40.20 & 24.67 & 22.09 & 37.78 & $24.6 \%$ & 22.51 & 23.22 & 1756 & 1601 & 27.71 & 2166 & 2108 & 4 & 14 \\
\hline Bष.33 & 39.15 & 23.30 & 20.38 & 36.54 & 23.38 & 21.11 & 26.24 & 16.72 & 1520 & 2639 & $20 \pi$ & 2006 & 9 & 2.1 \\
\hline $8 \times+6$ & 39.62 & 23.09 & 16.28 & 37.36 & 23.25 & 16.39 & 23.10 & 16.75 & $14.2 x$ & $2 \times 18$ & 21.07 & 1265 & 3 & 4.8 \\
\hline
\end{tabular}

On the other hand, PSNR values depend very much on image content. PSNR of image Lena is through all compression ratios for about $3 \mathrm{~dB}$ higher than PSNR for image Zebra. The difference between PSNR plots for DCT and DWT in Fig. 10(a) and (b) is much larger than the difference between PSNR plots in Fig. 10(c). The reason is different spectral activities of these three test images (Fig. 4). Image Zebra has high spectral activity. This type of image is less sensitive to the choice of compression method than images with low and moderate spectral activity. Compression performances for images with moderate spectral activity are sensitive to the choice of compression method. It is the reason image Lena is often used in the process of optimizing a compression system. The content of image Baboon is very difficult for the DCT coder to handle, especially for higher compression ratios [Fig. 10(d)]. The reason is that image Baboon contains a narrow range of luminance levels and a large number of details.

The comparison of PSNR and PQS values for a compression ratio below $10: 1$ is shown in Table VI. For the low compression ratios, $8 \times 8$ DCT and DWT show very similar characteristics (DCT produces slightly better results) for all images.

Measurements based on PQS cannot cover the wide range of compression ratios from $2: 1$ to $100: 1$. PQS quantifies some perceptual characteristics of a compression system, but PQS has some disadvantages. For higher compression ratios, PQS values turn out to be increasingly sharp, fall out of the valid range, and 
TABLE IV

PSNR AND PQS VALUES OF OPTIMAL WAVELETS FROM TABLE III FOR FIXED CRS

\begin{tabular}{|c|c|c|c|c|c|c|c|c|c|c|c|c|c|}
\hline \multicolumn{2}{|c|}{ Image } & \multicolumn{3}{|c|}{ Peppers } & \multicolumn{3}{|c|}{ Lena } & \multicolumn{3}{|c|}{ Zebra } & \multicolumn{3}{|c|}{ Baboon } \\
\hline$c a$ & Warest & DW.- & CW.S & $8 \times .21$ & DW-S & $\mathrm{CN}-\mathrm{s}$ & BW-22 & DWN-3 & $\mathrm{CW}-2$ & $10 \%-21$ & $D W-1$ & $\mathrm{CW} \cdot 3$ & घE. 22 \\
\hline \multirow{2}{*}{$2: 1$} & PSSNR & 49.42 & 49.09 & 49.92 & 47.01 & 47.05 & 47.46 & 36.65 & 36.57 & 35.57 & 35.76 & 36.63 & 35.53 \\
\hline & ros & 5.47 & 5.43 & 5.52 & 5.31 & 532 & 5,34 & 4.63 & 4.60 & 4.56 & 450 & 4,63 & 4.58 \\
\hline \multirow{2}{*}{$4: 1$} & P5NR & 41.53 & 41.65 & 42.27 & $39.0 \mathrm{t}$ & 3932 & 40.04 & 29.63 & 29.88 & 29,78 & 29.23 & 29.55 & 28.96 \\
\hline & POS & 4,30 & 4.28 & 4.52 & 4,47 & 451 & 4.68 & 3.51 & 3.56 & 3.68 & 3.17 & 3.17 & 3.19 \\
\hline \multirow{2}{*}{$8: 1$} & PSNR & 35,04 & 35.49 & 36.19 & 33.34 & 33.90 & 34.09 & 25.03 & 25.18 & 24.95 & 25.81 & 26.07 & 25.69 \\
\hline & $\mathrm{NOS}$ & 2.44 & 2.40 & 2.94 & 2.25 & 234 & 2.68 & 2.45 & 2.42 & 2.70 & 202 & 2.11 & 2.25 \\
\hline \multirow{2}{*}{$10: 1$} & FSNR & 33.34 & 33.72 & 34.43 & 31.74 & 32.17 & 32.51 & 23.75 & 23.90 & 23.58 & 25.03 & 25.24 & 24.91 \\
\hline & POS & 1.79 & 1.71 & 2.35 & I.64 & 1.78 & 2.18 & 2.19 & 2.19 & 2.52 & 1.78 & 1.82 & 1.88 \\
\hline
\end{tabular}

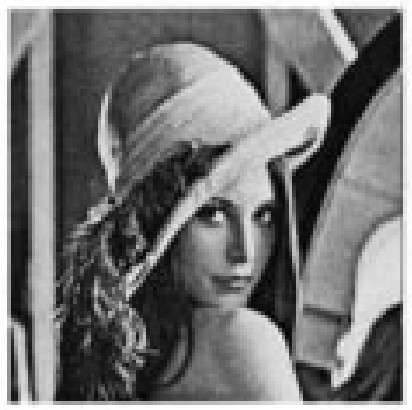

(a)

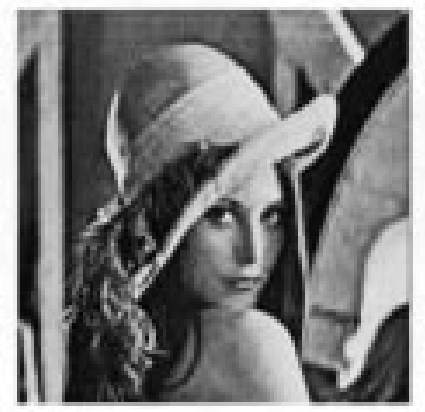

(b)

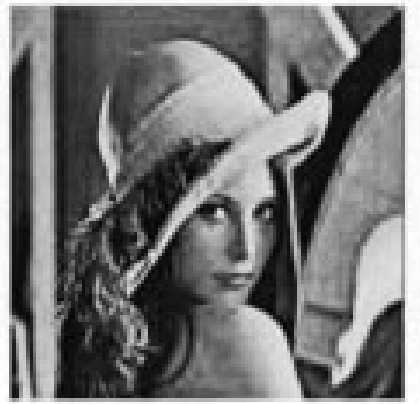

(c)

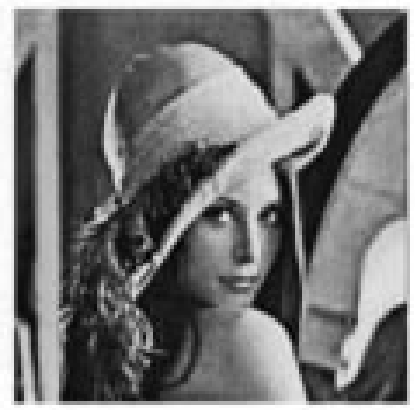

(d)

Fig. 8. Comparison of optimal wavelet functions for image Lena (PSNR $=36 \mathrm{~dB}$ ). (a) Original. (b) DW-5 (PQS = 2.93). (c) $\mathrm{CW}-3$ (PQS $=3.10)$. (d) BW-2.2 $(\mathrm{PQS}=3.20)$.

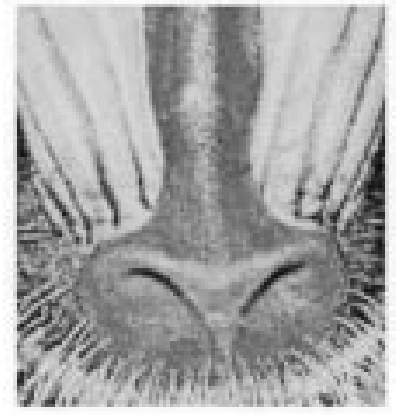

(a)

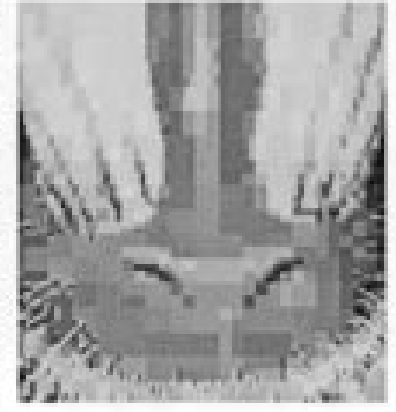

(b)

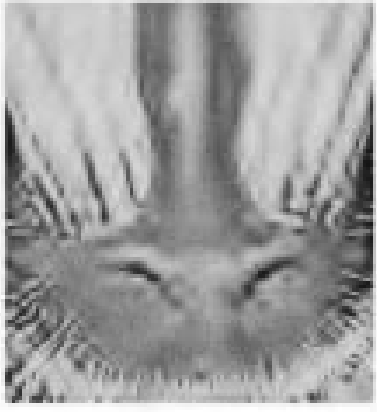

(c)

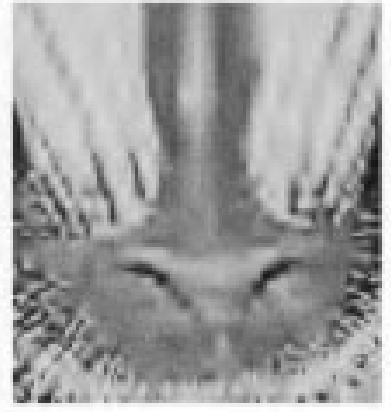

(d)

Fig. 9. Comparison of visual image quality for the detail from test image Baboon and fixed compression ratio 20:1. (a) Original. (b) DW-1. (c) CW-3. (d) BW-2.2.

become meaningless. Thus, we have to use subjective testing to complete results of visual image quality. The results of subjective measurements are contained in Table VII. Images are compressed using DWT and $8 \times 8$ DCT with four different compression ratios: $4: 1,10: 1,30: 1$, and $50: 1$ (we have to use a small number of compression ratios to reduce the time needed for subjective testing). MOS results show that human observers have more tolerance for moderately distorted images than PQS. The results are strongly influenced by image content. MOS includes psychological effects of the HVS that cannot be included in PQS. For HVS, the DWT coder works better than DCT at higher compression ratios for all types of images.

\section{CONCLUSIONS}

In this paper, we presented results from a comparative study of different wavelet-based image compression systems. The effects of different wavelet functions, filter orders, number of decompositions, image contents, and compression ratios are examined. The final choice of optimal wavelet in image compression application depends on image quality and computational complexity. We found that wavelet-based image compression prefers smooth functions of relatively short length. A suitable number of decompositions should be determined by means of image quality and less computational operation. Our results 
TABLE V

PSNR AND PQS VALUES OF 9/7 BW [13] AND BW-2.2

\begin{tabular}{c|c|c|c|c|c|c|c|c|c}
\hline \multicolumn{2}{c|}{ Image } & \multicolumn{2}{c|}{ Peppers } & \multicolumn{2}{c|}{ Lena } & \multicolumn{2}{c|}{ Zebra } & \multicolumn{2}{c}{ Baboon } \\
\hline \multirow{2}{*}{ CR } & Wavelet & $9 / 7 \mathrm{BW}$ & BW-2.2 & $9 / 7 \mathrm{BW}$ & $\mathrm{BW}-2.2$ & $9 / 7 \mathrm{BW}$ & $\mathrm{BW}-2.2$ & $9 / 7 \mathrm{BW}$ & $\mathrm{BW}-2.2$ \\
& & & & & & & & & \\
\hline \multirow{2}{*}{$\mathbf{2 : 1}$} & PSNR & 49.20 & 49.92 & 47.82 & 47.46 & 36.79 & 35.57 & 35.69 & 35.53 \\
& PQS & 5.50 & 5.53 & 5.48 & 5.34 & 4.55 & 4.56 & 4.45 & 4.58 \\
\hline \multirow{2}{*}{$4: 1$} & PSNR & 42.16 & 42.27 & 40.18 & 40.04 & 29.95 & 29.78 & 29.58 & 28.96 \\
& PQS & 4.40 & 4.52 & 4.35 & 4.68 & 3.52 & 3.68 & 3.11 & 3.19 \\
\hline \multirow{2}{*}{$\mathbf{8 : 1}$} & PSNR & 36.14 & 36.19 & 34.49 & 34.09 & 25.41 & 24.95 & 26.13 & 25.69 \\
& PQS & 2.63 & 2.94 & 2.45 & 2.68 & 2.47 & 2.70 & 2.09 & 2.25 \\
\hline \multirow{2}{*}{$\mathbf{1 0 : 1}$} & PSNR & 34.32 & 34.43 & 32.88 & 32.51 & 24.13 & 23.58 & 25.31 & 24.91 \\
& PQS & 1.97 & 2.35 & 2.05 & 2.18 & 2.27 & 2.52 & 1.79 & 1.88 \\
\hline \hline
\end{tabular}

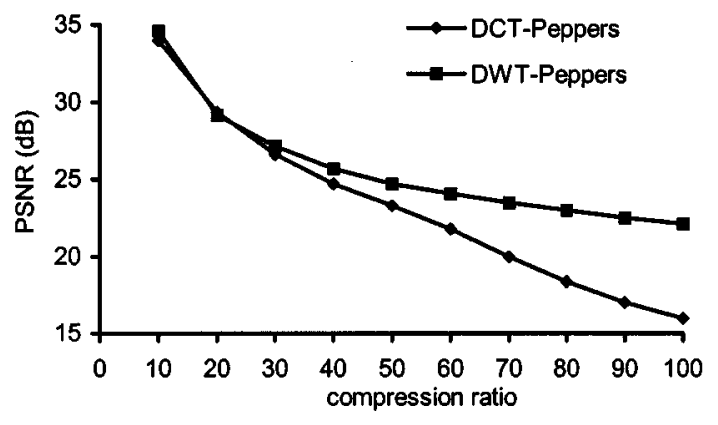

(a)

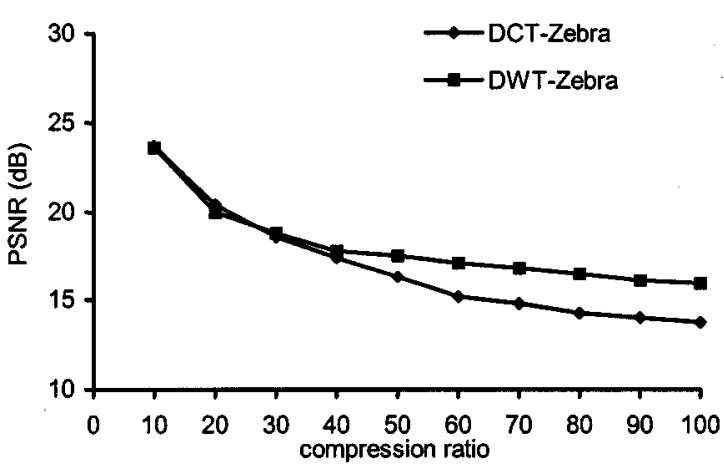

(c)

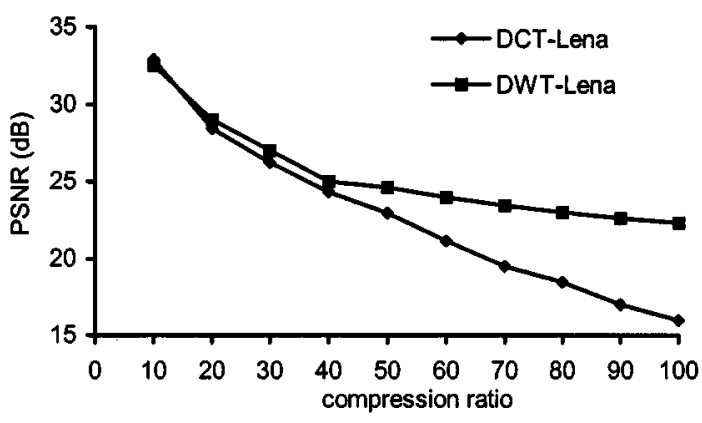

(b)

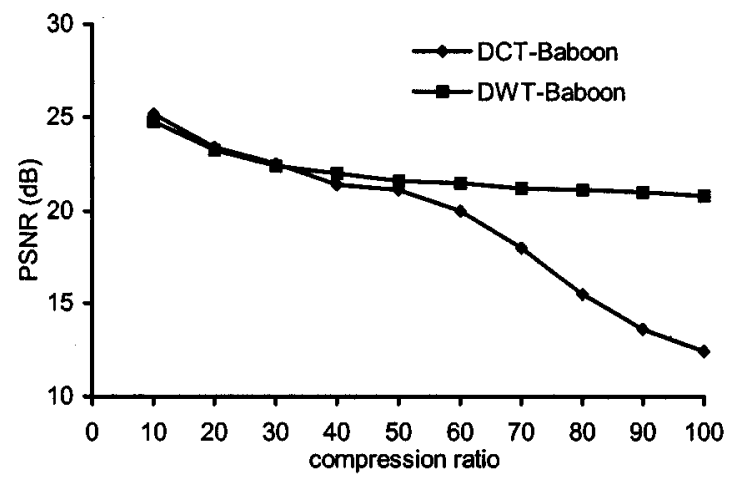

(d)

Fig. 10. Comparison of PSNR values for DCT and DWT of test images. (a) Peppers. (b) Lena. (c) Zebra. (d) Baboon.

show that the choice of optimal wavelet depends on the method, which is used for picture quality evaluation. We used objective and subjective picture quality measures. The objective measures such as PSNR and MSE do not correlate well with subjective quality measures. Therefore, we used PQS as an objective measure that has good correlation to subjective measurements. Our results show that different conclusions about an optimal wavelet can be achieved using PSNR and PQS. Each compression system should be designed with respect to the characteristics of the HVS. Therefore, our choice is based on PQS, which takes into account the properties of the HVS. We ana- lyzed results for a wide range of wavelets and found that BW-2.2 provides the best visual image quality for different image contents. Additionally, BW-2.2 has very low computational complexity in comparison with the other wavelets. BW-2.2 is used in analysis and comparison with DCT. Although DCT processing speed and compression capabilities are good, there are noticeable blocking artifacts at high compression ratios. However, DWT enables high compression ratios while maintaining good visual quality. Finally, with the increasing use of multimedia technologies, image compression requires higher performance as well as new features that can be provided using DWT. 

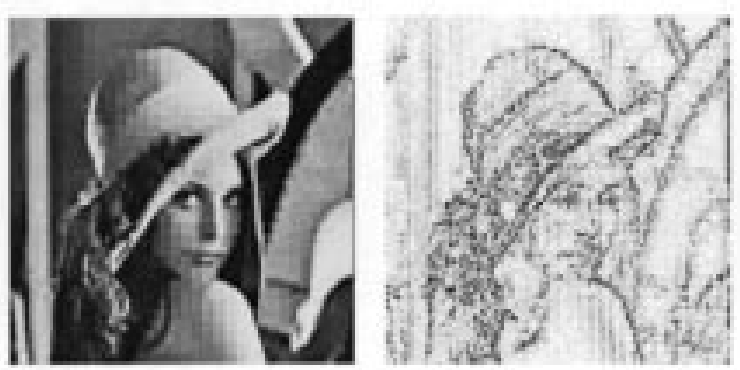

(a)
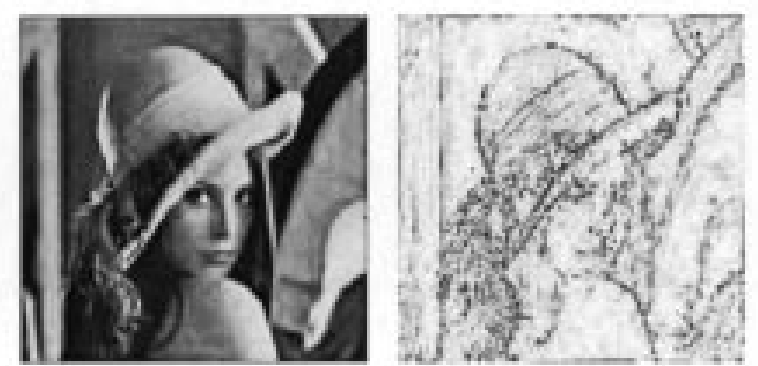

(b)

Fig. 11. Reconstructed image and reconstruction error for image Lena (PSNR = 26 dB). (a) DCT. (b) BW-2.2.

TABLE VI

PSNR AND PQS VALUES FOR COMPRESSION RATIO BELOW 10:1

\begin{tabular}{c|c|c|c|c|c|c|c|c|c}
\hline \multicolumn{2}{c|}{ Image } & \multicolumn{2}{c|}{ Peppers } & \multicolumn{2}{c|}{ Lena } & \multicolumn{2}{c|}{ Zebra } & \multicolumn{2}{c}{ Baboon } \\
\hline \multirow{2}{*}{ CR } & $\begin{array}{c}\text { Transform } \\
\text { Type }\end{array}$ & DCT & BW-2.2 & DCT & BW-2.2 & DCT & BW-2.2 & DCT & BW-2.2 \\
& & & & & & & & \\
\hline \multirow{2}{*}{$2: 1$} & PSNR & 49.90 & 49.92 & 47.70 & 47.46 & 36.80 & 35.57 & 36.50 & 35.53 \\
& PQS & 5.58 & 5.52 & 5.53 & 4.55 & 4.57 & 4.56 & 4.52 & 4.58 \\
\hline \multirow{2}{*}{$4: 1$} & PSNR & 42.02 & 42.27 & 40.05 & 40.04 & 29.5 & 29.78 & 29.51 & 28.96 \\
& PQS & 4.56 & 4.52 & 4.43 & 3.89 & 3.48 & 3.68 & 3.21 & 3.19 \\
\hline \multirow{2}{*}{$\mathbf{8 : 1}$} & PSNR & 36.04 & 36.19 & 34.40 & 34.09 & 25.00 & 24.95 & 26.12 & 25.69 \\
& PQS & 3.00 & 2.94 & 3.09 & 2.58 & 2.83 & 2.70 & 2.20 & 2.25 \\
\hline \multirow{2}{*}{$\mathbf{1 0 : 1}$} & PSNR & 34.30 & 34.43 & 32.9 & 32.51 & 23.71 & 23.58 & 25.30 & 24.91 \\
& PQS & 2.46 & 2.35 & 2.52 & 2.12 & 2.79 & 2.52 & 1.94 & 1.88 \\
\hline \hline
\end{tabular}

TABLE VII

MOS FOR $8 \times 8$ DCT AND DWT

\begin{tabular}{c|c|c|c|c|c|c|c|c}
\hline \hline Image & \multicolumn{2}{|c|}{ Peppers } & \multicolumn{2}{c|}{ Lena } & \multicolumn{2}{c|}{ Zebra } & \multicolumn{2}{c}{ Baboon } \\
\hline CR & DCT & BW-2.2 & DCT & BW-2.2 & DCT & BW-2.2 & DCT & BW-2.2 \\
\hline $\mathbf{4 : 1}$ & 5.00 & 5.00 & 5.00 & 5.00 & 5.00 & 5.00 & 5.00 & 5.00 \\
\hline $\mathbf{1 0 : 1}$ & 3.43 & 4.43 & 3.66 & 4.24 & 3.68 & 4.34 & 3.58 & 4.18 \\
\hline $\mathbf{3 0 : 1}$ & 2.12 & 3.04 & 1.94 & 2.65 & 2.36 & 2.45 & 2.08 & 2.56 \\
\hline $\mathbf{5 0 : 1}$ & 1.33 & 2.24 & 1.65 & 2.15 & 1.55 & 2.05 & 1.75 & 2.15 \\
\hline \hline
\end{tabular}

\section{REFERENCES}

[1] Proc. IEEE (Special Issue on Wavelets), vol. 84, Apr. 1996.

[2] N. Jayant and P. Noll, Digital Coding of Waveforms: Principles and Applications to Speech and Video. Englewood Cliffs, NJ: Prentice-Hall, 1984.

[3] N. Jayant, J. Johnston, and R. Safranek, "Signal compression based on models of human perception," Proc. IEEE, vol. 81, pp. 1385-1422, Oct. 1993.

[4] B. Zovko-Cihlar, S. Grgic, and D. Modric, "Coding techniques in multimedia communications," in Proc. 2nd Int. Workshop Image and Signal Processing, IWISP'95, Budapest, Hungary, 1995, pp. 24-32.

[5] Digital Compression and Coding of Continuous Tone Still Images, ISO/IEC IS 10918, 1991

[6] Information Technology-Coding of Moving Pictures and Associated Audio for Digital Storage Media at up to about $1.5 \mathrm{Mb} / \mathrm{s}$ : Video, ISO/IEC IS 11172, 1993.

[7] Information Technology_Generic Coding of Moving Pictures and Associated Audio Information: Video, ISO/IEC IS 13818, 1994.

[8] K. R. Rao and P. Yip, Discrete Cosine Transform: Algorithms, Advantages and Applications. San Diego, CA: Academic, 1990.
[9] S. Bauer, B. Zovko-Cihlar, and M. Grgic, "The influence of impairments from digital compression of video signal on perceived picture quality," in Proc. 3rd Int. Workshop Image and Signal Processing, IWISP'96, Manchester, U.K., 1996, pp. 245-248.

[10] A. S. Lewis and G. Knowles, "Image compression using the 2-D wavelet transform," IEEE Trans. Image Processing, vol. 1, pp. 244-250, Apr. 1992.

[11] M. L. Hilton, "Compressing still and moving images with wavelets," Multimedia Syst., vol. 2, no. 3, pp. 218-227, 1994.

[12] M. Antonini, M. Barland, P. Mathieu, and I. Daubechies, "Image coding using the wavelet transform," IEEE Trans. Image Processing, vol. 1, pp. 205-220, Apr. 1992.

[13] Z. Xiang, K. Ramchandran, M. T. Orchard, and Y. Q. Zhang, "A comparative study of DCT- and wavelet-based image coding," IEEE Trans. Circuits Syst. Video Technol., vol. 9, pp. 692-695, Apr. 1999.

[14] E. Feig, "A fast scaled DCT algorithm," Proc. SPIE-Image Process. Algorithms Techn., vol. 1244, pp. 2-13, Feb. 1990.

[15] J. M. Shapiro, "Embedded image coding using zerotrees of wavelet coefficients," IEEE Trans. Signal Processing, vol. 41, pp. 3445-3463, Dec. 1993.

[16] A. Said and W. A. Pearlman, "A new fast and efficient image codec based on set partitioning in hierarchical trees," IEEE Trans. Circuits Syst. Video Technol., vol. 6, pp. 243-250, June 1996. 
[17] I. Daubechies, Ten Lectures on Wavelets. Philadelphia, PA: SIAM, 1992.

[18] S. Mallat, "A theory of multiresolution signal decomposition: The wavelet representation," IEEE Trans. Pattern Anal. Machine Intell., vol. 11, pp. 674-693, July 1989.

[19] S. Mallat, "Multifrequency channel decomposition of images and wavelet models," IEEE Trans. Acoust., Speech, Signal Processing, vol. 37, pp. 2091-2110, Dec. 1989.

[20] - "Wavelets for a vision," Proc. IEEE, vol. 84, pp. 602-614, Apr. 1996.

[21] P. C. Cosman, R. M. Gray, and R. A. Olshen, "Evaluating quality of compressed medical images: SNR, subjective rating and diagnostic accuracy,” Proc. IEEE, vol. 82, pp. 920-931, June 1994.

[22] M. Ardito and M. Visca, "Correlation between objective and subjective measurements for video compressed systems," SMPTE J., pp. 768-773, Dec. 1996.

[23] J. Allnatt, Transmitted-Picture Assessment. New York: Wiley, 1983.

[24] M. Miyahara, K. Kotani, and V. R. Algazi. (1996, Aug.) Objective picture quality scale (PQS) for image coding. [Online]. Available: http://info.cipic.ucdavis.edu/scripts/reportPage?96-12

[25] "Methods for the subjective assessment of the quality of television pictures," ITU, Geneva, Switzerland, ITU-R Rec. BT. 500-7, Aug. 1998

[26] J. Lu, V. R. Algazi, and R. R. Estes, "Comparative study of wavelet image coders," Opt. Eng., vol. 35, pp. 2605-2619, Sept. 1996.

[27] M. Grgic, M. Ravnjak, and B. Zovko-Cihlar, "Filter comparison in wavelet transform of still images," in Proc. IEEE Int. Symp. Industrial Electronics, ISIE'99, Bled, Slovenia, 1999, pp. 105-110.

[28] S. Grgic, K. Kers, and M. Grgic, "Image compression using wavelets," in Proc. IEEE Int. Symp. Industrial Electronics, ISIE'99, Bled, Slovenia, 1999, pp. 99-104.

[29] W. R. Zettler, J. Huffman, and D. C. P. Linden, "Application of compactly supported wavelets to image compression," Proc. SPIE-Int. Soc. Opt. Eng., vol. 1244, pp. 150-160, 1990.

[30] A. Cohen, I. Daubechies, and J. C. Feauveau, "Biorthogonal bases of compactly supported wavelets," Comm. Pure Appl. Math., vol. 45, pp. 485-500, 1992

[31] A. B. Watson, Digital Images and Human Vision. Cambridge, MA MIT Press, 1993

[32] M. K. Mandal, S. Panchanathan, and T. Aboulnasr, "Choice of wavelets for image compression," Lecture Notes Comput. Sci., vol. 1133, pp. 239-249, 1996

[33] S. Grgic, M. Grgic, and B. Zovko-Cihlar, "Picture quality measurements in wavelet compression system," in Int. Broadcasting Convention Conf. Pub. IBC99, Amsterdam, The Netherlands, 1999, pp. 554-559.

[34] M. Mrak and N. Sprljan, "Discrete cosine transform in image coding applications," (in Croatian), Dept. Radiocommun. Microwave Eng., Univ. Zagreb, Internal Pub., 1999.

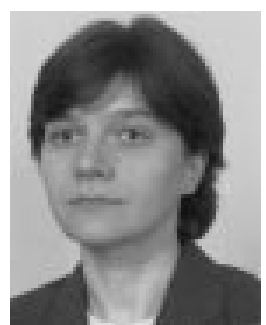

Sonja Grgic received the B.Sc., M.Sc., and Ph.D. degrees in electrical engineering from the Faculty of Electrical Engineering and Computing, University of Zagreb, Zagreb, Croatia, in 1989, 1992, and 1996, respectively.

She is currently an Assistant Professor in the Department of Radiocommunications and Microwave Engineering, Faculty of Electrical Engineering and Computing, University of Zagreb. Her research interests include television signal transmission and distribution, picture quality assessment, wavelet image compression, and broadband network architecture for digital television. She is a member of the international program and organizing committees of several international workshops and conferences. She was a Visiting Researcher in the Department of Telecommunications, University of Mining and Metallurgy, Krakow, Poland.

Prof. Grgic was the recipient of the Silver Medal "Josip Loncar" from the Faculty of Electrical Engineering and Computing, University of Zagreb, for outstanding Ph.D. dissertation work.

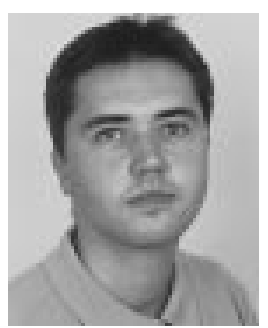

Mislav Grgic (S'96-A'96-M'97) received the B.Sc., M.Sc., and Ph.D. degrees in electrical engineering from the Faculty of Electrical Engineering and Computing, University of Zagreb, Zagreb, Croatia, in 1997,1998 , and 2000, respectively.

He is currently a Research Assistant in the Department of Radiocommunications and Microwave Engineering, Faculty of Electrical Engineering and Computing, University of Zagreb. His research interests include image and video compression, wavelet image coding, texture-based image retrieval, and digital video communications. He has been a member of the organizing committees of several international workshops and conferences. From October 1999 to February 2000, he conducted research in the Department of Electronic Systems Engineering, University of Essex, Colchester, U.K.

Dr. Grgic was the recipient of four Chancellor Awards for best student work, the Bronze Medal "Josip Loncar," for outstanding B.Sc. thesis work, and the Silver Medal "Josip Loncar" for outstanding M.Sc. thesis work from the Faculty of Electrical Engineering and Computing, University of Zagreb.

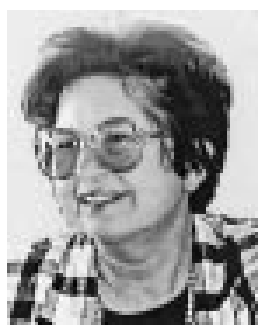

Branka Zovko-Cihlar (M'77) received the B.S. and $\mathrm{Ph} . \mathrm{D}$. degrees in electrical engineering from the Faculty of Electrical Engineering and Computing, University of Zagreb, Zagreb, Croatia, in 1959 and 1964, respectively.

She is currently a Professor in the Department of Radiocommunications and Microwave Engineering, Faculty of Electrical Engineering and Computing, University of Zagreb. From 1959 to 1961, she was with Radio-Industry Zagreb (RIZ), and from 1970 to 1984 , she worked intermittently at the LM Ericsson Laboratories. She has more than 35 years experience in television, broadcasting systems, and noise in radiocommunications. She is the author of Noise in Radiocommunications (Zagreb, Croatia: Skolska knjiga, 1987). She has been the Principal Investigator of four scientific projects. Presently, she is the Principal Investigator of the project "Multimedia Communication Technologies," financed by the Ministry of Science and Technology. She is a Coordinator of the international COST-237 and COST-257 Projects in Croatia. She is a member of the Editorial Board of the Journal of Electrical Engineering.

Prof. Zovko-Cihlar is the President of the Croatian Society of Electronics in Marine-ELMAR and a member of the Croatian Technical Academy. 